\title{
CONSTANT CURVATURE SURFACES IN A PSEUDO-ISOTROPIC SPACE
}

\author{
MUHITTIN EVREN AYDIN
}

\begin{abstract}
In this study, we deal with the local structure of curves and surfaces immersed in a pseudo-isotropic space $\mathbb{\unlhd}_{p}^{3}$ that is a particular Cayley-Klein space. We provide the formulas of curvature, torsion and Frenet trihedron for spacelike and timelike curves, respectively. The causal character of all admissible surfaces in $\mathbb{\sharp}_{p}^{3}$ has to be timelike up to its absolute. We introduce the formulas of Gaussian and mean curvature for timelike surfaces in $\rrbracket_{p}^{3}$. As applications, we describe the surfaces of revolution which are the orbits of a plane curve under a hyperbolic rotation with constant Gaussian and mean curvature.
\end{abstract}

\section{Introduction and preliminaries}

Let $P\left(\mathbb{R}^{3}\right)$ be the projective 3 -space and $\left(x_{0}: x_{1}: x_{2}: x_{3}\right)$ the homogenous coordinates. By a quadric, we mean a subset of points of $P\left(\mathbb{R}^{3}\right)$ described as zeros of a quadratic form associated with a non-zero symmetric bilinear form of $P\left(\mathbb{R}^{3}\right)$.

The Cayley-Klein 3-spaces can be defined in $P\left(\mathbb{R}^{3}\right)$ by an absolute figure, namely a sequence of quadrics and subspaces of $P\left(\mathbb{R}^{3}\right)$, see $[13,16,27,30]$. We are interested in a particular Cayley-Klein space, the pseudo-isotropic 3-space $\square_{p}^{3}$. Its absolute is composed of the quadruple $\left\{\omega, f_{1}, f_{2}, F\right\}$, where $\omega$ is the plane at infinity, $f_{1}, f_{2}$ two real lines in $\omega, F$ the intersection of $f_{1}$ and $f_{2}$. In coordinate form, these arguments are given by

$$
\omega: x_{0}=0, f_{1}: x_{0}=x_{1}=0, f_{2}: x_{0}=x_{2}=0, F(0: 0: 0: 1) .
$$

For further details, see $[9,14,18,19,29]$.

Our framework is to concern an affine model of $\mathbb{q}_{p}^{3}$ via the coordinates $\left(x=\frac{x_{1}}{x_{0}}, y=\frac{x_{2}}{x_{0}}, z=\frac{x_{3}}{x_{0}}\right), x_{0} \neq 0$. The group of pseudo-isotropic motions is a six-parameter group given by

$$
(x, y, z) \longmapsto\left(x^{\prime}, y^{\prime}, z^{\prime}\right):\left\{\begin{array}{l}
x^{\prime}=a+q x, \\
y^{\prime}=b+\frac{1}{q} y(q \neq 0), \\
z^{\prime}=c+d x+e y+z,
\end{array}\right.
$$

Received October 4, 2017, accpted March 15, 2018.

2010 Mathematics Subject Classification. 53A35, 53B25, 53B30, 53C42.

Key words and phrases. Pseudo-isotropic space, surface of revolution, Gaussian curvature, mean curvature. 
where $a, b, c, d, e, q \in \mathbb{R}$. The pseudo-isotropic metric is introduced by the absolute, i.e. $d s^{2}=$ $d x^{2}-d y^{2}$. Note that this metric can be also viewed as $d s^{2}=d x d y$ by standing $x=(x+y) / 2$, $y=(x-y) / 2$.

The investigation of curves and surfaces in 3-spaces is a classical field of study in differential geometry. In spite of the fact that the cyclides in $\mathbb{1}_{p}^{3}$, i.e. algebraic surfaces of order 4 , have been studied for many years; as far as we know, the local structure of curves and surfaces in $\rrbracket_{p}^{3}$ has not been established.

Indeed, we found motivation for this paper in B. Divjak's works ([10, 11, 22]), in which the differential geometry of curves and surfaces in the pseudo-Galilean space is introduced by generalizing that of the Galilean space. Intending a similar approach for the isotropic geometry, we are interested in the local theory of curves and surfaces in $\mathbb{q}_{p}^{3}$. For details of isotropic geometry, see [1]-[4], [12, 15], [23]-[25], [31].

The fact that the pseudo-isotropic metric is indefinite requires to introduce some basic notions (e.g. the causal character, the pseudo-angle, etc.) in $\mathbb{1}_{p}^{3}$ from the semi-Riemannian geometry (see Section 2). For detailed properties of such a geometry see $[6,17,26]$.

In Section 3, we show that each lightlike curve in $\rrbracket_{p}^{3}$ lies in the isotropic plane of the form $x \pm y=c, c \in \mathbb{R}$. As the local structures of the non-lightlike curves, the formulas in $\square_{p}^{3}$ analogous to the well-known Frenet's formulas were given.

It is suprisingly observed in Section 4 that each immersed admissible surface in $\rrbracket_{p}^{3}$ is timelike. The formulas of the Gaussian and the mean curvatures for timelike surfaces are also introduced.

As several applications, in Section 5, we study and classify the surfaces of revolution, imposing some natural curvature conditions.

\section{Basics in the sense of pseudo-isotropic geometry}

Let $(x, y, z)$ be the isotropically orthogonal system in $\mathbb{q}_{p}^{3}$. For some vector $u \in \mathbb{q}_{p}^{3}$, let us consider the projection onto $x y$-plane given by

$$
u=\left(u_{1}, u_{2}, u_{3}\right) \longmapsto \tilde{u}=\left(u_{1}, u_{2}, 0\right)
$$

usually called top view. The pseudo-isotropic scalar product between two vectors $u=\left(u_{1}, u_{2}, u_{3}\right)$, $v=\left(v_{1}, v_{2}, v_{3}\right) \in \mathbb{\mathbb { R }}_{p}^{3}$ can be defined as their Lorentzian scalar product in top view, namely $\langle u, v\rangle=u_{1} v_{1}-u_{2} v_{2}$.

A line is said to be isotropic (resp. non-isotropic) if its point at infinity is (resp. no) the absolute point $F$. Moreover, a plane is said to be isotropic (resp. non-isotropic) if its line at infinity contains (resp. does not) the absolute point $F$. In the affine model of $\square_{p}^{3}$, the isotropic 
lines and planes are parallel to the $z$-axis. In the non-isotropic planes, the Lorentzian metric is basically used.

A nonzero vector $u$ is said to be isotropic (resp. non-isotropic) if $\tilde{u}=0$ (resp. $\tilde{u} \neq 0$ ). The zero vector is assumed to be non-isotropic. A non-isotropic vector $u \in \mathbb{q}_{p}^{3}$ is respectively called spacelike, timelike and lightlike (or null) if $\langle u, u\rangle>0$ or $u=0,\langle u, u\rangle<0$ and $\langle u, u\rangle=0(u \neq 0)$.

The set of all lightlike vectors of $\square_{p}^{3}$ is called lightlike cone, i.e.,

$$
\Lambda=\left\{\left(u_{1}, u_{2}, u_{3}\right) \in \mathbb{\square}_{p}^{3} \mid u_{1}^{2}-u_{2}^{2}=0\right\}-\left\{0 \in \mathbb{\square}_{p}^{3}\right\}
$$

Denote $\mathscr{T}$ the set of all timelike vectors in $\mathbb{q}_{p}^{3}$. For some $u \in \mathscr{T}$, the set given by

$$
\mathscr{C}(u)=\{v \in \mathscr{T}:\langle u, v\rangle<0\}
$$

is called the timelike cone of $\square_{p}^{3}$ containing $u$.

The pseudo-isotropic angle of two timelike non-isotropic vectors $u, v \in \mathbb{Q}_{p}^{3}$ lying in the same timelike-cone is defined as the Lorentzian angle between $\tilde{u}$ and $\tilde{v}$, i.e.

$$
\langle u, v\rangle=-\sqrt{-\langle u, u\rangle} \sqrt{-\langle\nu, v\rangle} \cosh \phi .
$$

Note that all isotropic vectors are isotropically orthonogal to non-isotropic ones. Further, two non-isotropic vectors $u, v$ in $\rrbracket_{p}^{3}$ are orthonogal if $\langle u, v\rangle=0$.

\section{Spacelike and timelike curves in $\rrbracket_{p}^{3}$}

Let $\alpha(s)=(x(s), y(s), z(s))$ be a regular curve in $\mathbb{q}_{p}^{3}$, i.e. $\alpha^{\prime}(s)=\frac{d \alpha}{d s} \neq 0$ for all $s$. Then it is said to be admissible if $\alpha(s)$ has no isotropic osculating plane. An admissible curve $\alpha(s)$ in $\mathbb{1}_{p}^{3}$ is said to be spacelike (resp. timelike, lightlike) if $\alpha^{\prime}(s)$ is spacelike (resp. timelike, lightlike) for all $s$.

An easy computation shows that all lightlike curves lie in the isotropic plane of the form $x \pm y=c, c \in \mathbb{R}$.

Henceforth, we are only interested in spacelike and timelike admissible curves.

Now let $\alpha=\alpha(s)$ be a spacelike curve in $\rrbracket_{p}^{3}$ parameterized by arc-length $s$. Then we have

$$
\left\langle\alpha^{\prime}, \alpha^{\prime}\right\rangle=x^{\prime 2}(s)-y^{\prime 2}(s)=1
$$

for all $s$. Taking derivative of (3.1) gives

$$
x^{\prime}(s) x^{\prime \prime}(s)-y^{\prime}(s) y^{\prime \prime}(s)=0 .
$$


Introduce $\mathbf{t}(s)=\alpha^{\prime}(s)$ and call it tangent vector of $\alpha$ at $s$. Because $\mathbf{t}^{\prime}(s)=\alpha^{\prime \prime}(s)$ is timelike in $\mathbb{q}_{p}^{3}$ we can define the following

$$
\kappa=\sqrt{y^{\prime \prime 2}(s)-x^{\prime \prime 2}(s)}
$$

called curvature of $\alpha$ at $s$. Using (3.2), we get

$$
\kappa(s)=\frac{y^{\prime \prime}(s)}{x^{\prime}(s)} \text { or } \kappa=\frac{x^{\prime \prime}(s)}{y^{\prime}(s)},\left(x^{\prime}(s) y^{\prime}(s) \neq 0\right) .
$$

Considering (3.1) and (3.2) into (3.3) we find

$$
\kappa(s)=\operatorname{det}\left(\tilde{\alpha}^{\prime}(s), \tilde{\alpha}^{\prime \prime}(s)\right) .
$$

Let the normal vector and torsion of $\alpha$ at $s$ be introduced by, respectively

$$
\mathbf{n}(s)=\frac{1}{\kappa(s)} \mathbf{t}^{\prime}(s) \text { and } \tau(s)=\frac{\operatorname{det}\left(\alpha^{\prime}(s), \alpha^{\prime \prime}(s), \alpha^{\prime \prime \prime}(s)\right)}{\kappa^{2}(s)}, \kappa(s) \neq 0 .
$$

Because $\mathbf{b}(s)=(0,0,1)$ is isotropically orthogonal to both $\mathbf{t}(s)$ and $\mathbf{n}(s)$, we can take it as the binormal vector of $\alpha$ at $s$. From (3.5) we have

$$
\mathbf{n}^{\prime}(s)=\left(\frac{1}{\kappa(s)}\right)^{\prime}\left(x^{\prime \prime}(s), y^{\prime \prime}(s), z^{\prime \prime}(s)\right)+\frac{1}{\kappa(s)}\left(x^{\prime \prime \prime}(s), y^{\prime \prime \prime}(s), z^{\prime \prime \prime}(s)\right) .
$$

Introduce $\mathbf{n}^{\prime}(s)=\left(n_{1}(s), n_{2}(s), n_{3}(s)\right)$. Hence we write

$$
n_{1}(s)=\left(\frac{1}{\kappa(s)}\right)^{\prime} x^{\prime \prime}(s)+\frac{1}{\kappa(s)} x^{\prime \prime \prime}(s) .
$$

Using (3.4) into (3.7) yields

$$
n_{1}(s)=-\frac{x^{\prime}(s)}{\kappa^{2}(s)}\left(x^{\prime \prime}(s) y^{\prime \prime \prime}(s)-x^{\prime \prime \prime}(s) y^{\prime \prime}(s)\right) .
$$

By taking derivative of (3.2) and then considering into (3.8) we obtain

$$
n_{1}(s)=\kappa(s) x^{\prime}(s) .
$$

Similar computations gives

$$
n_{2}(s)=\kappa(s) y^{\prime}(s) .
$$

For the third component of $\mathbf{n}^{\prime}$, we have

$$
n_{3}(s)=\left(\frac{1}{\kappa(s)}\right)^{\prime} z^{\prime \prime}(s)+\frac{1}{\kappa(s)} z^{\prime \prime \prime}(s) .
$$

It follows from (3.4) that

$$
n_{3}=\frac{1}{\kappa^{2}}\left\{-\left(x^{\prime} y^{\prime \prime \prime}-x^{\prime \prime \prime} y^{\prime}\right) z^{\prime \prime}+\left(x^{\prime} y^{\prime \prime}-x^{\prime \prime} y^{\prime}\right) z^{\prime \prime \prime}\right\} .
$$


By adding and substracting $\left(x^{\prime \prime}(s) y^{\prime \prime \prime}(s)-y^{\prime \prime}(s) x^{\prime \prime \prime}(s)\right) z^{\prime}(s)$ in (3.11) we conclude

$$
n_{3}(s)=\frac{1}{\kappa^{2}(s)}\left\{\operatorname{det}\left(\alpha^{\prime}(s), \alpha^{\prime \prime}(s), \alpha^{\prime \prime \prime}(s)\right)-\left(x^{\prime \prime}(s) y^{\prime \prime \prime}(s)-x^{\prime \prime \prime}(s) y^{\prime \prime}(s)\right) z^{\prime}(s)\right\} .
$$

Taking derivative of (3.2) and considering into (3.12) implies

$$
n_{3}(s)=\tau(s)+\kappa(s) z^{\prime}(s) .
$$

(3.9), (3.10) and (3.13) conclude that $\mathbf{n}^{\prime}(s)=\kappa(s) \mathbf{t}(s)+\tau(s) \mathbf{b}(s)$. Thus we obtain the formulas analogous to these of Frenet as follows

$$
\frac{d}{d s}\left(\begin{array}{l}
\mathbf{t} \\
\mathbf{n} \\
\mathbf{b}
\end{array}\right)=\left(\begin{array}{lll}
0 & \kappa & 0 \\
\kappa & 0 & \tau \\
0 & 0 & 0
\end{array}\right)\left(\begin{array}{l}
\mathbf{t} \\
\mathbf{n} \\
\mathbf{b}
\end{array}\right)
$$

By similar arguments, we can find the Frenet's formulas for a timelike curve in $\mathbb{q}_{p}^{3}$ as

$$
\frac{d}{d s}\left(\begin{array}{l}
\mathbf{t} \\
\mathbf{n} \\
\mathbf{b}
\end{array}\right)=\left(\begin{array}{ccc}
0 & \kappa & 0 \\
\kappa & 0 & -\tau \\
0 & 0 & 0
\end{array}\right)\left(\begin{array}{l}
\mathbf{t} \\
\mathbf{n} \\
\mathbf{b}
\end{array}\right)
$$

where

$$
\kappa(s)=\sqrt{x^{\prime \prime 2}(s)-y^{\prime \prime 2}(s)}=-\operatorname{det}\left(\tilde{\alpha}^{\prime}(s), \tilde{\alpha}^{\prime \prime}(s)\right) \text { and } \tau=\frac{\operatorname{det}\left(\alpha^{\prime}(s), \alpha^{\prime \prime}(s), \alpha^{\prime \prime \prime}(s)\right)}{\kappa^{2}(s)} .
$$

Example 3.1. Let a spacelike curve of arc-length in $\rrbracket_{p}^{3}$ be given by

$$
\alpha(s)=\frac{1}{2}\left(s^{2}, s \sqrt{s^{2}-1}-\ln \left|s+\sqrt{s^{2}-1}\right|, 2 s\right)
$$

for $s \in\left(1, \frac{3 \pi}{2}\right)$. Its curvature and torsion writes $\kappa(s)=-\tau(s)=\frac{1}{\sqrt{s^{2}-1}}$. Notice that such a curve can be viewed as a general helix because the ratio $\frac{\tau(s)}{\kappa(s)}$ is a constant. In addition, it is easy to see that one satisfies the Frenet's formulas. We plot it as in Figure 1.

Example 3.2. Let us consider a hyperbolic cylindrical curve (see [7]) in $\rrbracket_{p}^{3}$ given by

$$
\alpha(s)=(\cosh s, \sinh s, z(s)) .
$$

We plot it as in Figure 2. This is a timelike curve of arc-length in $\rrbracket_{p}^{3}$ with $\kappa(s)=1$ and

$$
\tau(s)=z^{\prime}(s)-z^{\prime \prime \prime}(s) .
$$

It is clear that one satisfies the Frenet's formulas. In addition, if $\alpha$ has constant torsion $\tau_{0}$, then by solving (3.16) we find

$$
z(s)=\tau_{0} s+c_{1} e^{s}-c_{2} e^{-s}+c_{3}, c_{1}, c_{2}, c_{3} \in \mathbb{R},
$$

which gives the following elemantary result: 
Proposition 3.1. Let $\alpha$ be a hyperbolic cylindrical curve in $\square_{p}^{3}$ with constant torsion $\tau_{0}$. Then it is of the form

$$
\alpha(s)=\left(\cosh s, \sinh s, \tau_{0} s+c_{1} e^{s}-c_{2} e^{-s}+c_{3}\right),
$$

where $c_{1}, c_{2}, c_{3} \in \mathbb{R}$.

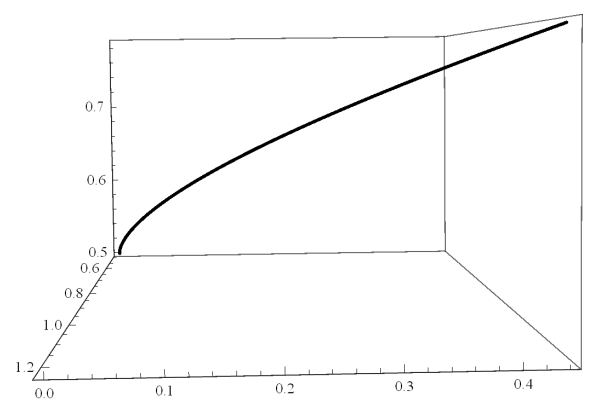

Figure 1: A spacelike general helix with $\frac{\tau(s)}{\kappa(s)}=-1, s \in(1,3 \pi / 2)$.

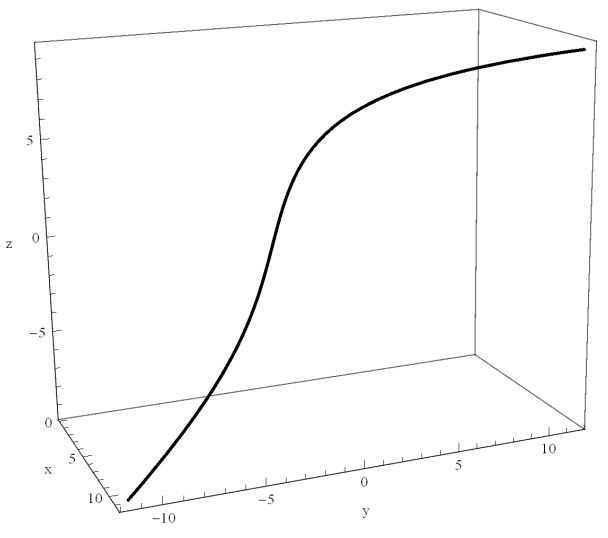

Figure 2: A hyperbolic cylindrical curve with $\tau_{0}=3, s \in(-\pi, \pi)$.

\section{Timelike surfaces in $\rrbracket_{p}^{3}$}

Let $M$ be a surface immersed in $\mathbb{q}_{p}^{3}$ without isotropic tangent planes. Then we call such a surface admissible. Assume that $M$ has a local parameterization in $\mathbb{\square}_{p}^{3}$ as follows:

$$
\mathbf{r}: D \subseteq \mathbb{R}^{2} \longrightarrow \mathbb{1}_{p}^{3},\left(u_{1}, u_{2}\right) \longmapsto\left(x\left(u_{1}, u_{2}\right), y\left(u_{1}, u_{2}\right), z\left(u_{1}, u_{2}\right)\right)
$$

for smooth real-valued functions $x, y, z$ on a domain $D \subseteq \mathbb{R}^{2}$. Let $g$ be the induced metric on $M$ from $\square_{p}^{3}$ and denote $\left(g_{i j}\right)$ its matrical expression with respect to the basis $\left\{\mathbf{r}_{u_{1}}, \mathbf{r}_{u_{2}}\right\}$. Then we have

$$
g_{i j}=\left\langle\mathbf{r}_{u_{i}}, \mathbf{r}_{u_{j}}\right\rangle, \mathbf{r}_{u_{i}}=\frac{\partial \mathbf{r}}{\partial u_{i}}, i, j=1,2 .
$$


It is easy to see that

$$
\operatorname{det}\left(g_{i j}\right)=-\left(x_{u_{1}} y_{u_{2}}-x_{u_{2}} y_{u_{1}}\right)^{2} .
$$

Here the admissibility assures $\operatorname{det}\left(g_{i j}\right) \neq 0$. In other words, one concludes that admissible surfaces are all timelike.

The unit normal vector field of $M$ is the isotropic vector $\xi=(0,0,1)$ because it is isotropically orthogonal to the tangent plane of $M$ at each point.

In order to introduce the second fundamental form of $M$, we follow the similar way with Sachs (see [28, p. 155]). Let $\mathbf{r}(s)$ be an arc-length curve on $M$ and $\mathbf{t}$ its tangent vector. We can take a side tangential vector $\sigma$ in the tangent plane of $M$ such that $\{\mathbf{t}, \sigma\}$ is a positive oriented base. Therefore we have a decomposition:

$$
\mathbf{r}^{\prime \prime}=\frac{d^{2} \mathbf{r}}{d s^{2}}=\kappa \mathbf{n}=\kappa_{g} \sigma+\kappa_{n} \xi,
$$

where $\mathbf{n}, \kappa_{g}$ and $\kappa_{n}$ are the normal vector, geodesic and normal curvatures of $\mathbf{r}$ on $M$, respectively. Put $\sigma=a_{1} \mathbf{r}_{u_{1}}+a_{2} \mathbf{r}_{u_{2}}$. Because $\langle\mathbf{t}, \sigma\rangle=0$ and $\mathbf{t}=\mathbf{r}_{u_{1}} \frac{d u_{1}}{d s}+\mathbf{r}_{u_{2}} \frac{d u_{2}}{d s}$, we get

$$
a_{1}=\theta\left(g_{12} \frac{d u_{1}}{d s}+g_{22} \frac{d u_{2}}{d s}\right), a_{2}=-\theta\left(g_{11} \frac{d u_{1}}{d s}+g_{12} \frac{d u_{2}}{d s}\right),
$$

where $\theta=\theta\left(u_{1}, u_{2}\right)$ is some nonzero smooth function. Then we achieve

$$
1=\operatorname{det}(\tilde{\mathbf{t}}, \tilde{\sigma})=-\sqrt{\left|\operatorname{det}\left(g_{i j}\right)\right|} \theta
$$

and hence

$$
\sigma=-\frac{1}{\sqrt{\left|\operatorname{det}\left(g_{i j}\right)\right|}}\left[\left(g_{12} \frac{d u_{1}}{d s}+g_{22} \frac{d u_{2}}{d s}\right) \mathbf{r}_{u_{1}}-\left(g_{11} \frac{d u_{1}}{d s}+g_{12} \frac{d u_{2}}{d s}\right) \mathbf{r}_{u_{2}}\right] .
$$

Accordingly, we compute that

$$
\begin{aligned}
\kappa_{n} & =\operatorname{det}\left(\mathbf{r}^{\prime}, \sigma, \mathbf{r}^{\prime \prime}\right)=\frac{1}{\sqrt{\left|\operatorname{det}\left(g_{i j}\right)\right|}} \operatorname{det}\left(\mathbf{r}_{u_{1}}, \mathbf{r}_{u_{2}}, \mathbf{r}^{\prime \prime}\right) \\
& =\frac{1}{\sqrt{\left|\operatorname{det}\left(g_{i j}\right)\right|}} \sum_{i, j=1}^{2} \operatorname{det}\left(\mathbf{r}_{u_{1}}, \mathbf{r}_{u_{2}}, \mathbf{r}_{u_{i} u_{j}}\right)\left(\frac{d u_{i}}{d s}\right)\left(\frac{d u_{j}}{d s}\right),
\end{aligned}
$$

which leads to the components of the second fundamental form given by

$$
h_{i j}=\frac{\operatorname{det}\left(\mathbf{r}_{u_{1}}, \mathbf{r}_{u_{2}}, \mathbf{r}_{u_{i} u_{j}}\right)}{\sqrt{\left|\operatorname{det}\left(g_{i j}\right)\right|}}, r_{u_{i} u_{j}}=\frac{\partial^{2} \mathbf{r}}{\partial u_{i} \partial u_{j}}, i, j=1,2 \text {. }
$$

Thus the Gaussian curvature and the mean curvature of $M$ are respectively defined by

$$
K=\frac{\operatorname{det}\left(h_{i j}\right)}{\operatorname{det}\left(g_{i j}\right)}
$$


and

$$
H=\frac{g_{11} h_{22}-2 g_{12} h_{12}+g_{22} h_{11}}{2 \operatorname{det}\left(g_{i j}\right)} .
$$

By permutation of the coordinates, two different types of graph surfaces appear up to the absolute of $\square_{p}^{3}$. For a graph of the function $u=u(x, y)$, the formulas (4.1) and (4.2) reduce to

$$
K=-u_{x x} u_{y y}+\left(u_{x y}\right)^{2}, H=\frac{1}{2}\left(u_{x x}-u_{y y}\right) .
$$

Because the metric on the graph surface induced from $\square_{p}^{3}$ is $g=d x^{2}-d y^{2}$, it always becomes a flat surface. So, its Laplacian turns to

$$
\triangle=\frac{\partial^{2}}{\partial u_{1}^{2}}-\frac{\partial^{2}}{\partial u_{2}^{2}}
$$

On the other side, the Gaussian and mean curvatures of the graph of $u=u(y, z)$ are given by

$$
K=-\frac{u_{y y} u_{z z}-\left(u_{y z}\right)^{2}}{\left(u_{z}\right)^{4}}, \quad H=\frac{\left(u_{z}\right)^{2} u_{y y}-2 u_{y} u_{z} u_{y z}+\left(\left(u_{y}\right)^{2}-1\right) u_{z z}}{2\left(u_{z}\right)^{3}},
$$

where $u_{z} \neq 0$ due to the admissibility.

\section{Constant curvature surfaces of revolution in $\mathbb{q}_{p}^{3}$}

Da Silva [8] provided via hyperbolic numbers that the pseudo isotropic motion given by $\bar{x}=q x, \bar{y}=\frac{1}{q} y, q \neq 0$ is equivalent to the hyperpolic rotation (about $z$-axis) given by

$$
\bar{x}=x \cosh \theta+y \sinh \theta, \bar{y}=x \sinh \theta+y \cosh \theta,
$$

where $\theta \in \mathbb{R}$.

Let $u \longmapsto(u, 0, f(u))$ be a spacelike admissible curve lying in the isotropic $x z$-plane of $\square_{p}^{3}$ for a smooth function $f$. Rotating it around $z$-axis via hyperbolic rotations given by (5.1) we derive

$$
\mathbf{r}(u, v)=(u \cosh v, u \sinh v, f(u)) .
$$

We call the rotating curve profile curve. If the profile curve is a timelike curve $u \longmapsto(0, u, f(u))$ lying in the isotropic $y z$-plane of $\square_{p}^{3}$, then rotating it around $\mathbf{z}$-axis yields

$$
\mathbf{r}(u, v)=(u \sinh v, u \cosh v, f(u)) .
$$

The surfaces given by (5.2) and (5.3) are called surfaces of revolution in $\mathbb{q}_{p}^{3}$. The Gaussian curvature of these surfaces in $\square_{p}^{3}$ is

$$
K=\frac{f^{\prime} f^{\prime \prime}}{u},
$$


where $f^{\prime}(u)=\frac{d f}{d u}$, etc.

Now we assume that it has nonzero constant Gaussian curvature $K_{0}$ in $\rrbracket_{p}^{3}$. Then (5.4) can be rewritten as

$$
f^{\prime}=\sqrt{c_{1}+K_{0} u^{2}}, c_{1} \in \mathbb{R}
$$

After integrating (5.5), we obtain

$$
f(u)=\frac{u}{2} \sqrt{c_{1}+K_{0} u^{2}}+\frac{c_{1}}{2 \sqrt{K_{0}}} \ln \left(2 K_{0} u+2 \sqrt{K_{0}} \sqrt{c_{1}+K_{0} u^{2}}\right)+c_{2}, c_{2} \in \mathbb{R}
$$

which implies the following result.

Theorem 5.1. Let $M$ be a surface of revolution in $\rrbracket_{p}^{3}$ with nonzero constant Gaussian curvature $K_{0}$. Then its profile curve is of the form $(u, 0, f(u))$, where

$$
f(u)=\frac{u}{2} \psi(u)+\frac{c_{1}}{2 \sqrt{K_{0}}} \ln \left|2 \sqrt{K_{0}}\left(\sqrt{K_{0}} u+\psi(u)\right)\right|+c_{2}
$$

$\operatorname{for} \psi(u)=\sqrt{c_{1}+K_{0} u^{2}}, c_{1}, c_{2} \in \mathbb{R}$.

From (5.4), we immediately have the following.

Corollary 5.1. A surface of revolution is flat in $\rrbracket_{p}^{3}$ if and only if its profile curve is a non-isotropic line given by $\left(u, 0, c_{1} u+c_{2}\right), c_{1}, c_{2} \in \mathbb{R}$.

The mean curvature $H$ of a surface of revolution $M$ in $\rrbracket_{p}^{3}$ is

$$
H=\frac{1}{2}\left(\frac{f^{\prime}}{u}+f^{\prime \prime}\right)
$$

Assume that $M$ has constant mean curvature $H_{0}$. After solving (5.6) we deduce

$$
f(u)=\frac{H_{0}}{2} u^{2}+c_{1} \ln |u|+c_{2}, c_{1}, c_{2} \in \mathbb{R} .
$$

Therefore we have proved the following results.

Theorem 5.2. Let $M$ be a surface of revolution in $\rrbracket_{p}^{3}$ with constant mean curvature $H_{0}$. Then its profile curve is of the form $(u, 0, f(u))$, where

$$
f(u)=\frac{H_{0}}{2} u^{2}+c_{1} \ln |u|+c_{2}, c_{1}, c_{2} \in \mathbb{R} .
$$

Corollary 5.2. A surface of revolution is minimal in $\square_{p}^{3}$ if and only if its profile curve is a nonisotropic curve given by $\left(u, 0, c_{1} \ln u+c_{2}\right), c_{1}, c_{2} \in \mathbb{R}$. 
Example 5.1. Let the surfaces of revolution in $\rrbracket_{p}^{3}$ be given by

$$
\mathbf{r}(u, v)=(u \cosh v, u \sinh v, u),(u, v) \in[1,2] \times[0,1]
$$

and

$$
\mathbf{r}(u, v)=\left(u \cosh v, u \sinh v, \ln u+u^{2}\right),(u, v) \in[1,2] \times[-1,1] .
$$

The above first surface is flat and the second is a constant mean curvature surface of revolution, $H=2$. We plot these as in Figure 3 and Figure 4, respectively.

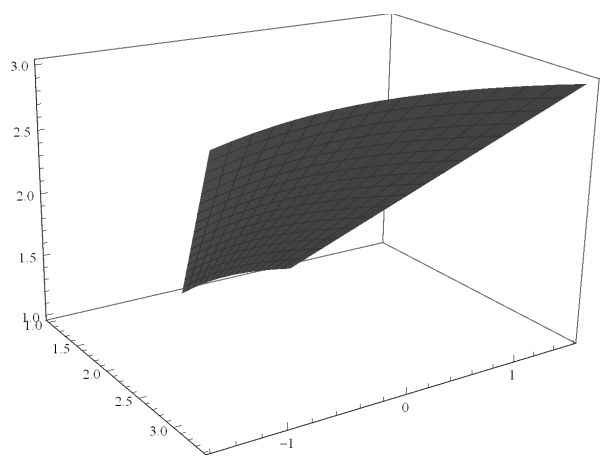

Figure 3: A flat surface of revolution, $K=0$.

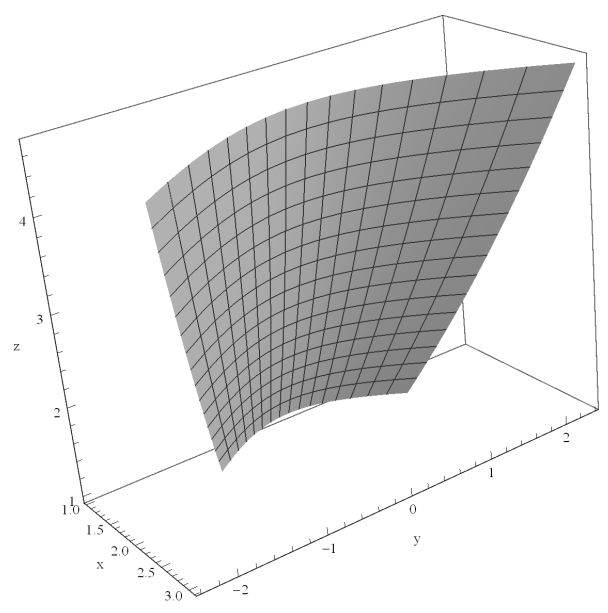

Figure 4: A constant curvature surface of revolution, $H=2$.

6. Surfaces of revolution with $H^{2}=K$ in $\mathbb{q}_{p}^{3}$

Next we aim to classify the surfaces of revolution given by (5.2) in $\rrbracket_{p}^{3}$ that satisfy $H^{2}=K$ which is the equality sign of the Euler inequality. For more generalizations of the well-known inequality, see [5, 20, 21]. 
By considering (5.4) and (5.6), we have

$$
\frac{1}{4}\left(\left(\frac{f^{\prime}}{u}\right)^{2}+2 \frac{f^{\prime} f^{\prime \prime}}{u}+\left(f^{\prime \prime}\right)^{2}\right)=\frac{f^{\prime} f^{\prime \prime}}{u}
$$

We can rewrite (6.1) as

$$
\left(\frac{f^{\prime}}{u}-f^{\prime \prime}\right)^{2}=0
$$

which implies

$$
\frac{f^{\prime}}{u}-f^{\prime \prime}=0
$$

After solving this, we obtain

$$
f(u)=c_{1} \frac{u^{2}}{2}+c_{2}
$$

for $c_{1}, c_{2} \in \mathbb{R}$. By comparing (5.2) with (6.2) we see that the surface of revolution can be given in explicit form

$$
z=\frac{c_{1}}{2}\left(x^{2}-y^{2}\right)+c_{2},
$$

which implies the following result.

Theorem 6.1. The surfaces of revolution given by (5.2) in $\rrbracket_{p}^{3}$ with $H^{2}=K$ are only the spheres of parabolic type.

Example 6.1. Consider the sphere of parabolic type in $\mathbb{\square}_{p}^{3}$ given by (6.3) such that $c_{1}=2$ and $c_{2}=0$. Then its Gaussian and mean curvatures become $H=2$ and $K=4$. We plot it as in Figure 5.

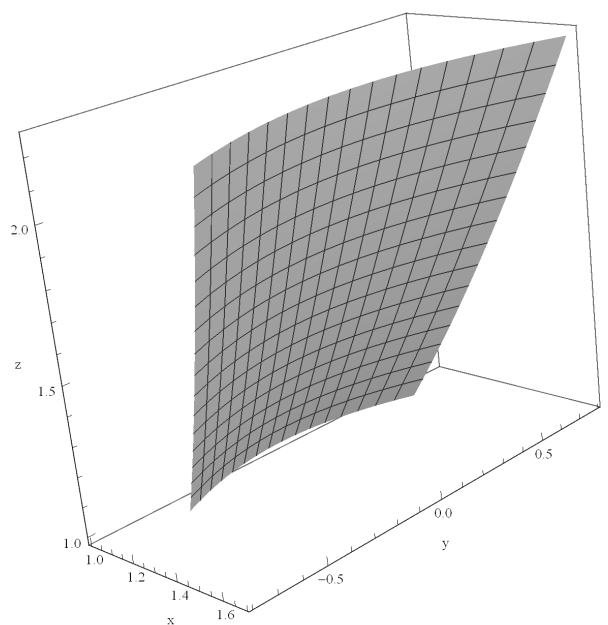

Figure 5: A surface of revolution with $H^{2}=K$. 


\section{Acknowledgements}

The author thanks specially to L.C.B. Da Silva (Universidade Federal de Pernambuco) for his valuable discussions on the present study. The figures in this study were made by Wolfram Mathematica 11.0.

\section{References}

[1] M. E. Aydin and A. Mihai, Ruled surfaces generated by elliptic cylindrical curves in the isotropic space, Georgian Math. J. 2017; doi.org/10.1515/gmj-2017-0044.

[2] M. E. Aydin and I. Mihai, On certain surfaces in the isotropic 4-space, Math. Commun. 22(1) (2017), 41-51.

[3] M. E. Aydin, Classification results on surfaces in the isotropic 3-space, AKU J. Sci. Eng., 16(2016), $239-246$.

[4] B. Y. Chen, S. Decu and L. Verstraelen, Notes on isotropic geometry of production models, Kragujevac J. Math., 38(1) (2014), 23-33.

[5] B. Y. Chen, Mean curvature and shape operator of isometric immersions in real-space-forms, Glasg. Math. J., 38 (1996), 87-97.

[6] B. Y. Chen, Pseudo-Riemannian geometry, $\delta$-Invariants and applications, World Scientific, Singapore, 2011.

[7] M. Crasmareanu, Cylindrical Tzitzeica curves implies forced harmonic oscillators, Balkan J. Geom. Appl., 7(1)(2002), 37-42.

[8] L. C. B. Da Silva, Rotation minimizing frames and spherical curves in simply isotropic and semi-isotropic 3spaces, arXiv:1707.06321v2 [math.DG].

[9] L. C. B. Da Silva, The geometry of Gauss map and shape operator in simply isotropic and pseudo-isotropic spaces, arXiv:1801.01187v1 [math.DG].

[10] B. Divjak, Geometrija pseudogalilejevih prostora (Ph.D. thesis), University of Zagreb, 1997.

[11] B. Divjak, Curves in pseudo-Galilean geometry, Annales Universitatis Scientiarum Budapestinensis de Rolando Eötvös Nominatae, 41 (1998), 117-128.

[12] Z. Erjavec, B. Divjak and D. Horvat, The general solutions of Frenet's system in the equiform geometry of the Galilean, pseudo-Galilean, simple isotropic and double isotropic space, Int. Math. Forum 6(17) (2011), 837856.

[13] O. Giering, Vorlesungen über höhere Geometrie, Friedr. Vieweg \& Sohn, Braunschweig, Germany, 1982.

[14] M. Husty and O. Röschel, On a particular class of cyclides in isotropic respectively pseudoisotropic space, Coll. Math. Soc. J. Bolyai, 46 (1984), 531-557.

[15] M. K. Karacan, D. W. Yoon and S. Kiziltug, Helicoidal surfaces in the three dimensional simply isotropic space $I_{1}^{3}$, Tamkang J. Math., 48 (2017), 123-134.

[16] D. Klawitter, Clifford Algebras: Geometric Modelling and Chain Geometries with Application in Kinematics, Springer Spektrum, 2015.

[17] R. Lopez, Differential Geometry of curves and surfaces in Lorentz-Minkowski space, Int. Electron. J. Geom., 7(2014), 44-107.

[18] F. Meszaros, Die Zykliden 3. Ordnung im pseudoisotropen Raum II, Math. Pannonica 4(2)(1993), $273-285$.

[19] F. Meszaros, Klassifikationstheorie der verallgemeinerten zykliden 4. ordnung in pseudoisotropen Raum, Math. Pannonica, 18(2)(2007), 299-323.

[20] A. Mihai, Geometric inequalities for purely real submanifolds in complex space forms, Results Math., $\mathbf{5 5}$ (2009), 457-468.

[21] I. Mihai, On the generalized Wintgen inequality for Lagrangian submanifolds in complex space forms, Nonlinear Analysis 95 (2014), 714-720.

[22] Z. Milin-Sipus and B. Divjak, Surfaces of constant curvature in the pseudo-Galilean space, Int. J. Math. Sci., 2012, Art ID375264, 28pp. 
[23] Z. Milin-Sipus, Translation surfaces of constant curvatures in a simply isotropic space, Period. Math. Hung., 68 (2014), 160-175.

[24] A. O. Ogrenmis, Rotational surfaces in isotropic spaces satisfying Weingarten conditions, Open Physics 14(9) (2016), 221-225.

[25] A. O. Ogrenmis, M. Bektas and M. Ergut, On helices in the doubly isotropic space $I_{3}^{(2)}$, Int. Math. Forum 1 (13) (2006), 623-627.

[26] B. O‘Neill, Semi-Riemannian Geometry with Applications to Relativity, Academic Press, New York, 1983.

[27] A. Onishchick, R. Sulanke, Projective and Cayley-Klein Geometries, Springer, 2006.

[28] H. Sachs, Isotrope Geometrie des Raumes, Vieweg Verlag, Braunschweig, 1990.

[29] K. Strubecker, Differentialgeometrie des isotropen Raumes III, Flachentheorie, Math. Zeitsch., 48 (1942), 369 427.

[30] I. M. Yaglom, A simple non-Euclidean Geometry and Its Physical Basis, An elementary account of Galilean geometry and the Galilean principle of relativity, Heidelberg Science Library. Translated from the Russian by Abe Shenitzer. With the editorial assistance of Basil Gordon. Springer-Verlag, New York-Heidelberg, 1979.

[31] D.W. Yoon, J.W. Lee, Linear Weingarten helicoidal surfaces in isotropic space, Symmetry 2016, 8(11), 126; doi:10.3390/sym8110126.

Department of Mathematics, Faculty of Science, Firat University, Elazig, 23119, Turkey.

E-mail: meaydin@firat.edu.tr 